See discussions, stats, and author profiles for this publication at: https://www.researchgate.net/publication/312005617

\title{
Commodity Video Game Technology in Teletherapy
}

Chapter · August 2017

DOI: 10.1007/978-981-4560-50-4_26

CITATIONS

0

3 authors:

Gary Ushaw

Newcastle University

33 PUBLICATIONS 188 CITATIONS

SEE PROFILE

Graham Morgan

Newcastle University

85 PUBLICATIONS 756 CITATIONS

SEE PROFILE
59

Richard Davison

Newcastle University

11 PUBLICATIONS 48 CITATIONS

SEE PROFILE 


\title{
Commodity Video Game Technology in Teletherapy
}

\author{
Gary Ushaw, Richard Davison, Graham Morgan \\ School of Computing Science \\ Newcastle University
}

\section{Introduction}

The diversity and widespread adoption of domestic gaming systems has led to much interest in utilising action video games in the field of teletherapy. The availability of motion detection devices for these systems has further increased interest in utilising commodity video game technology in rehabilitation of impaired dexterity.

Rehabilitative gaming has a number of benefits. Firstly, the game should provide a more involving context, so that the patient enjoys the rehabilitative process and is more likely to be engaged in it. Secondly, the exercises can easily be carried out at home as gaming systems which include motioncapture devices are relatively commonplace and affordable. Finally, data on the patient's progress and recovery can be collected as the software monitors the patient's actions. The suitability of domestic gaming systems to meet the first two requirements of engagement and accessibility seem intuitively feasible. However the choice of device, and analysis of the input to meet the fidelity requirements of data collection for medical investigation and monitoring, requires discussion at the outset of any rehabilitative gaming project.

Many motion capture devices are available for domestic use, including Microsoft Kinect, Nintendo Wiimote Plus, Sony PS Move and Sixense Hydra. In this chapter a benchmark for domestic motion capture devices is described, and used to measure and compare the fidelity of the data and the reliability of the systems. The results should serve as a recommendation for future projects utilising domestic gaming devices for rehabilitation.

Further to the choice of gaming system most suited to a teletherapy project, many choices must be made during the design and development of a rehabilitative game to ensure that the patient is sufficiently engaged to allow for meaningful monitoring of progress and rehabilitation. The second part of this chapter discusses one such rehabilitative game, describing the choices made in its implementation based on tenets and knowledge from both the wider games industry and medical practitioners. The game was designed by a team of professional game developers and medical experts. The discussion is intended to provide a framework of recommendations to be considered when applying serious gaming to the field of teletherapy.

\section{Motion Capture Devices}

Motion capture devices have become a commonplace aspect of commercial gaming systems. Such devices are provided, either as a standard feature of the system, or as an affordable add-on, by all three of the major gaming console manufacturers (Nintendo, Sony and Microsoft). There are also 
motion gaming devices readily available for use with personal computers (such as the Sixense Hydra). This profusion of motion tracking systems has led to their successful employment in many rehabilitative studies. In this section, the functionality of each of the widely available devices is described in turn. The compatibility of the devices with PC technology is also addressed, as teletherapy projects are more likely to be developed for personal computer than console (due to the accessibility of the hardware and SDKs).

\section{Nintendo Wii MotionPlus}

Nintendo were first to market with a motion sensing device for a domestic gaming console. The technology proved disruptive to the games industry's accepted noms, achieving huge success for the Wii console with family audiences. The Wii system consists of a sensor bar (to be placed on or under the television set), and one or more handheld remote (or Wiimote). There are two aspects contributing to the system's motion sensing ability. Firstly the sensor bar has an infra-red LED cluster at each side, and the Wiimote motion controllers contain an infra-red camera for tracking their position relative to the sensor bar. Secondly, the controllers each contain three-axis accelerometers and three-axis gyroscopes which are used for dead-reckoning calculation of position, orientation and velocity. An official SDK for utilising Wiimote technology with a PC has not been released. However, bespoke software can be written which intercepts and interprets the data-stream from the devices via a Bluetooth wireless connection.

\section{Sony PlayStation Move}

The PlayStation Move from Sony consists of one or more hand-held controller (or "wand"), and a RGB camera to be mounted on the television or monitor. At the top of each wand is an illuminated sphere. The camera tracks this sphere in three dimensions; the distance from the camera is measured from the size of the sphere in the image. Each wand also contains a three-axis accelerometer, thee-axis gyroscope and a geomagnetic sensor. Consequently the system can track both the position and orientation of the wand. The geomagnetic sensor is used to calibrate the measurements against the earth's magnetic field, thereby addressing cumulative errors from the other sensors. The combination of camera tracking and dead-reckoning are intended to provide motion tracking of the wand whether it is visible to the camera or not. The system knows what colour the wand sphere is illuminated with, so the image processing software is searching for a circular shape of a known colour (a much less computationally intensive activity than searching an image for body parts or non-illuminated controllers). This means that the latency is much less than in other systems where more complex calculations are required. A potential drawback of applying the PlayStation Move to domestic rehabilitation is that the official SDK (the "Move.Me") requires the use of a PlayStation3 as a server, in addition to the PC that is running the application. This could lead to an unwieldy set-up for home use.

\section{Microsoft Kinect and Kinect 2}

Microsoft's Kinect motion sensing system directly monitors the user's body, so no hand-held devices are required. The system consists of a sensor bar which contains a RGB camera, an infra-red laser pattern projector and an infra-red camera. Three dimensional points in space for each bone of a humanoid skeleton are triangulated using the stereo pair of the infra-red projector and camera. Three images are made available by the system: colour, infra-red image, and an interpolated inverse depth image. Positional information for each bone in the detected skeletal model is made available 
by the Kinect SDK. The orientation of the bones can then be calculated from the joint hierarchy. Depending on the lighting conditions, and the complexity of the image, the system delivers motion tracking information for up to 20 skeletal joints per user at up to 30 frames per second (Smisek 2013).

A second generation of Kinect device was released for Microsoft's Xbox One in 2013 (and for Windows8 in 2014). The updated system incorporates a wider field of vision camera, capable of calculating the position and orientation of 25 joints per user, as well as their heart rate and facial expression. The colour map resolution has been increased, and the depth map has been augmented by time-of-flight technology contained in the new camera hardware affording a unique depth value per pixel. The combination of this technology and resolution should result in more accurate tracking as compared to the original Kinect device. Both Kinect and Kinect2 are supported via official SDK for the Windows environment, making it an easily accessibly device for development of PC based rehabilitative applications.

\section{Sixense}

Sixense Entertainment have introduced a motion tracking device for use with PC applications, which uses magnetic field motion tracking to provide continuous position and orientation information. This is in contrast to the camera and infra-red based systems developed by the console manufacturers. The use of electromagnetic fields is a well-established technology for reliably measuring threedimensional space (Hansen 1987). Devices which are reliant on cameras are prone to interruption due to line-of-sight issues; such problems do not occur with magnetic tracking. The Sixense comprises of a base unit, which is connected to the PC via USB, and wireless controllers which the user holds while performing the movements. The base unit contains three orthogonally orientated magnetic coils which emit an electromagnetic field, providing the reference for the position and orientation of the controllers. Similarly each controller contains three smaller orthogonal coils whose position and orientation are measured relative to the emitter's coils. Consequently each controller broadcasts three-dimensional position and orientation data to the base unit. The use of three controllers, one in each hand, and one tucked into the belt or pocket at waist level, provides further data on the patient's base position and motion. The device and the SDK are designed to be utilised with PC hardware.

\section{Benchmarking the Devices}

At the outset of a project in rehabilitative gaming a vital decision which must be made. Identifying the most appropriate motion capture device to utilise is of paramount importance. Each available device has performance advantages and disadvantages. This section describes a benchmark for assessing these devices in terms of the range and fidelity of captured movement data. Results are presented from carrying out the benchmarking test, leading to recommendations on which devices are most suitable to the particular demands of different rehabilitative projects.

\section{The Experiment}

Three easily defined and easily repeated movements are used as the benchmarks of this study. The first benchmark movement is to move the hand in a vertical circle, at full arm's length, in front of the subject (i.e. a circle in the coronal plane). The second is to hold the arm at full length in front of the subject and turn 360 degrees on the spot, so that the hand moves in a horizontal circle at shoulder 
height around the subject's position (i.e. a circle in the transverse plane). The third involves the subject swinging the arm in a vertical circle, keeping the arm at full length (i.e. a circle in the sagittal plane). A number of different subjects were used in the testing of each device. The devices must be able to cope consistently with patients of various shapes and sizes if they are to be used successfully in rehabilitation.

Markers were placed at 1 metre intervals up to 4 metres, with the sensing device placed at a height of $120 \mathrm{~cm}$. Users stood with their heels inline to each of these markers in turn. At each distance, the user stretched their arm fully forward, and rotated it fully 360 degree in each axis - for the coronal and sagittal planes, this took the form of solely shoulder rotation, while the transverse plane necessitated the user to rotate their entire body using their legs and feet, while keeping their back straight. For each plane, the rotation was performed three times, with the second rotation used to calculate results, in order to reduce side effects from the user speeding up, slowing down, or otherwise moving the controllers differently as the trial started and came to an end.

\section{Metrics}

Each device calculates 3D position and orientation in some way, so it is desirable to measure the quality of both. For a measurement of positional accuracy, only the relevant two axes for each plane from each trial-run are considered, creating a sequence of $2 \mathrm{D}$ coordinates that represent the vertices of a $2 \mathrm{D}$ polygon. Each trial's data is then centred on the $2 \mathrm{D}$ origin, according to the centroid of the polygon, calculated from all $n$ positions $p$ in the sequence $s$ as:

$$
\operatorname{Centroid}(s)=\frac{p_{0}+p_{1}+\cdots+p_{n}}{n}
$$

Once the captured data has been transformed in this way, two metrics are determined by which to rate the quality of the data: circularity and orientation drift. The circularity of a data set $s$ is defined using the following common shape factor (where $a$ is the area of the shape and $p$ its perimeter):

$$
\operatorname{Circularity}(s)=\frac{4 \pi a}{p^{2}}
$$

As the coordinates are two dimensional, the area and perimeter of the polygon can be calculated using the Shoelace formula:

$$
\begin{gathered}
\operatorname{Area}(s)=\frac{1}{2}\left|\sum_{i=1}^{n-1} p_{i} x p_{i+1} y+p_{n} x+p_{1} y-\sum_{i=1}^{n-1} p_{i} y p_{i+1} x+p_{n} y+p_{1} x\right| \\
\text { Perimeter }(s)=\sum_{i=1}^{n-1}\left\|\overrightarrow{p_{i+1}-p_{i}}\right\|+\left\|\overrightarrow{p_{n}-p_{1}}\right\|
\end{gathered}
$$

The accuracy of orientation is calculated in a similar way. By transforming the relevant forward basis vector for the input device by an orientation from the recorded sequence, a vector pointing in the direction of the device is created. By projecting this vector onto the plane being tested, a 2D position is formed. For directions that lie exactly on the plane, the point lies at a distance of 1.0. Deviations in the direction away from the plane result in projected points closer to the origin. The projected direction vectors over the course of one movement form a 2D polygon that are tested using the 
circularity measurement described previously. This method does not take into account deviations in rotation around the axis, but still serves as a useful metric of orientation quality.

As the circularity calculation provides values in the range $[0,1]$, the circularity of position and orientation can be used to calculate a measure of data quality as a simple arithmetic mean. Finally, a base of expected noise is measured, by recording a 30 second sample of the device at rest. The standard deviation in position on all three axes, and cosine of angle difference from the forward basis vector is then calculated.

A sequence of 3D positions $p$ and orientations $q$ are recorded, with timestamps of each sample. Positions are converted from the internal metric of the device to metres, while orientations are stored as quaternions. Orientations are transformed such that the local-space 'forward' reference frame of the device points along the user's outstretched arm - this is necessary as the Wii MotionPlus uses a coordinate system where the z-axis is 'up', rather than 'forward'.

The Kinects are entirely camera based. This results in a lack of information when the camera cannot see the user's hand. The PS Move uses camera tracking for positional data, so it too suffers from a drop-out in positional information when the Move 'wand' is occluded. Although orientation can still be tracked from the sensors contained within the Move device, for the purposes of this experiment they were ignored when the position was not determinable, to preserve the 1:1 mapping of positions to orientations. This is expected to be most evident when recording movements in the transverse plane, as the user's body obscures the camera's view of the hand / wand, with some additional occlusion expected with the sagittal plane recordings. While any loss of data is undesirable, the ability for the devices to redetect and track the desired movement after obscuration is a useful metric when considering the efficacy of motion devices.

\section{Steadiness}

Steadiness measurements were made at a distance of $2 \mathrm{~m}$. Table 1 shows the sum of the changes between the samples in a 30s recording of the device at rest, and the change between the first and last samples. Immediately obvious is the poor result in movement from the Wii Remote Plus - the sum distance is several orders of magnitude larger than any other, due to accumulated error in double integration of position from acceleration. Both the MoveMe and Sixense have large sum distances recorded, but, due to the low change in final position, it can be inferred that this was due to a high frequency jitter, rather than a large drift over time. The Wii Remote Plus reports an acceleration value without any processing for gravity; this was accounted for by taking a short sample of the accelerometer vector at rest, and subtracting this value, transformed by the orientation of the device. Evidently this was not sufficient to reliably remove gravity from the accelerometer reading, and further processing would be required to create a stable result.

\begin{tabular}{|c|c|c|c|c|}
\hline Device & Sum Distance & Sum Angle & $\begin{array}{c}\text { Distance } \\
\text { Travelled }\end{array}$ & Angle Travelled \\
\hline Kinect 1 & 0.0842 & 0,693 & 0.00369 & 0.00961 \\
\hline Kinect 2 & 0.954 & 149.878 & 0.000908 & 0.719 \\
\hline PS Move & 2101.93 & 8.835 & 8.944 & 0.00169 \\
\hline Sixense & 227.936 & 1.219 & 4.271 & 0.00825 \\
\hline MotionPlus & 4281.13 & 2.254 & 4272.98 & 0.202 \\
\hline
\end{tabular}

Table 1: Device steadiness comparison 
The Kinect2 shows a large accumulated sum angle, despite a low distance travelled. As the orientation is determined from the skeleton (calculated by the Kinect software) this is a curious result, as it suggests that the 'parent' joint of the skeletal hierarchy has had a large drift or jitter in its position. The low distances for the Kinect devices are at odds with the other tested devices, suggesting additional filtering of position. The low changes in angle over time compared to position for all devices is a good indicator of the relative difficulties in determining these values.

\section{Data Sample Rate}

The devices communicate with the host PC in a variety of ways: Kinect via direct USB connection, Sixense wirelessly to a USB connected base unit, and PS Move via Bluetooth to a PlayStation3 running the MoveMe server, which then contacts the host computer via TCP/IP. Due to this, and the differing ways in which position and orientation are derived, it is useful to compare update rates as reported from the interface software. The mean sample counts for each device across all recorded trial runs are collated in Table 2.

\begin{tabular}{|c|c|c|c|}
\hline Device & Coronal & Transverse & Sagittal \\
\hline Kinect 1 & 70.125 & 117.0 & 56.625 \\
\hline Kinect 2 & 36.381 & 75.864 & 34.9 \\
\hline PS MoveMe & 140.583 & 220.231 & 144.333 \\
\hline Sixense & 137.667 & 188.267 & 142.933 \\
\hline
\end{tabular}

Table 2: Sample counts for each device

The Kinect devices update at a significantly lower rate than the MoveMe and Sixense devices, which both report at a similar rate. The effects of this are discussed later.

\section{Data Circularity}

The circularity metric calculations for each device are shown in Figure 1. The results from each device are discussed in more detail in this section.

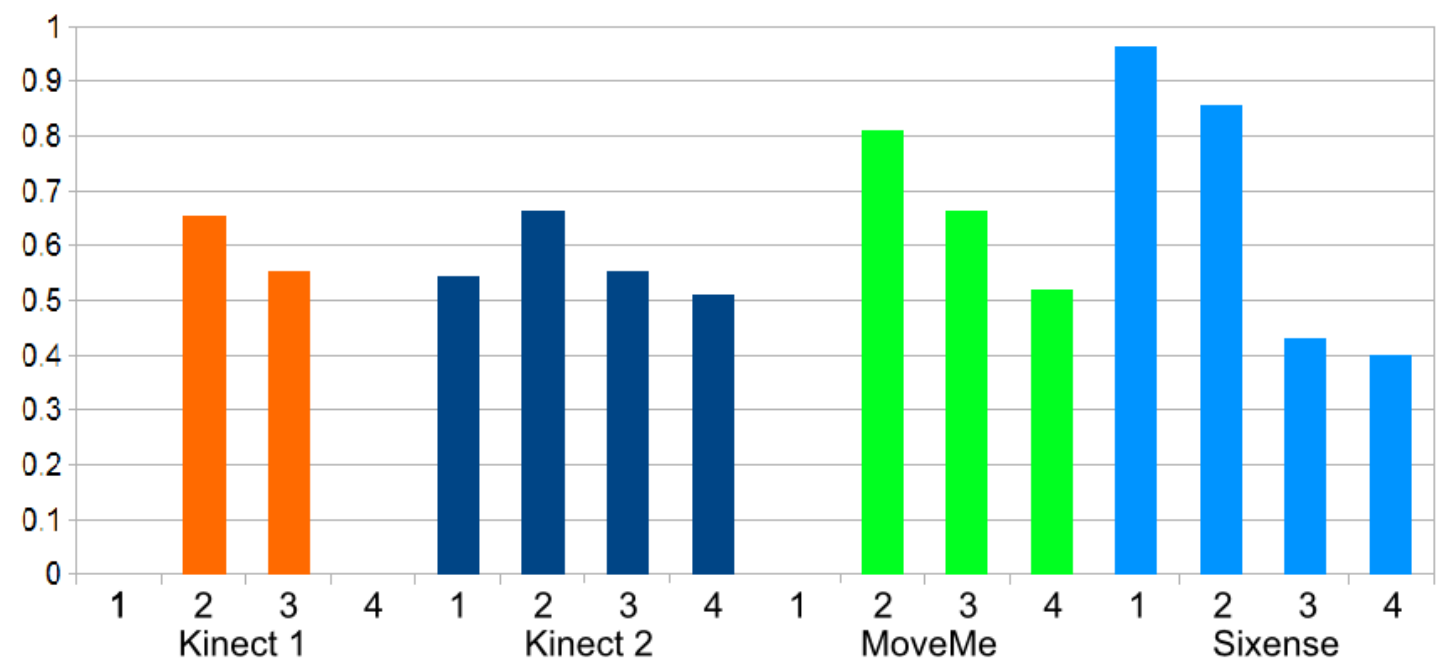

Figure 1: Device circularity comparison at distances of $1 \mathrm{~m}, 2 \mathrm{~m}, 3 \mathrm{~m}$ and $4 \mathrm{~m}$.

\begin{tabular}{|l|c|c|c|c|}
\hline Device & Distance $(\mathbf{m})$ & Orientation & Position & Quality \\
\hline Kinect 1 & 1 & - & - & - \\
\hline
\end{tabular}




\begin{tabular}{|l|c|c|c|c|}
\hline \multirow{4}{*}{} & 2 & 0.7048 & 0.6035 & 0.6542 \\
\cline { 2 - 5 } & 3 & 0.5124 & 0.5958 & 0.5541 \\
\cline { 2 - 5 } & 4 & - & - & - \\
\cline { 2 - 5 } & 1 & 0.5294 & 0.5283 & 0.5289 \\
\cline { 2 - 5 } & 2 & 0.6558 & 0.6849 & 0.6703 \\
\cline { 2 - 5 } & 3 & 0.5392 & 0.5555 & 0.5474 \\
\hline \multirow{4}{*}{ PS Move } & 4 & 0.5181 & 0.4888 & 0.5034 \\
\cline { 2 - 5 } & 1 & - & - & - \\
\cline { 2 - 5 } & 2 & 0.9752 & 0.6458 & 0.8105 \\
\hline \multirow{4}{*}{ Sizense } & 3 & 0.9409 & 0.3863 & 0.6636 \\
\cline { 2 - 5 } & 1 & 0.7634 & 0.2417 & 0.5025 \\
\cline { 2 - 5 } & 2 & 0.9805 & 0.9487 & 0.9646 \\
\cline { 2 - 5 } & 3 & 0.8749 & 0.8354 & 0.8552 \\
\hline
\end{tabular}

Table 3: Device circularity comparison. Distances are measured in metres (dashes indicates distances for which no data could be recorded for that device)

\section{Wii MotionPlus}

The position of the Wii MotionPlus device can be determined in two ways: Either via double integration of its accelerometer data over time, or by processing the position of the IR light sources from the tracker bar, as seen by the Wii's onboard camera (broadly, the closer together the points, the further away the device is from the tracker bar). Unfortunately, the experiment as designed is unsuitable for position determination via the tracker bar, as the bar will never be in the line of sight when rotating in the coronal plane, and only briefly when rotating in the transverse or sagittal planes. Accurate derivation of position from accelerometer data is notoriously difficult, as any noise or bias in the accelerometer data will quickly accumulate into large errors in position. As gravity will be detected by the accelerometers, it must be accounted for, and any inaccuracy in this calculation will again result in large errors in position. As gravity will be detected by the accelerometers, it must be accounted for, and any inaccuracy in this calculation will again result in large errors in position.

Despite the poor positional output, a test trial of rotating in the transverse plane shows excellent stability, as shown in a plot in Figure 2, of the $x$ and $z$ components of the transformed forward vectors of each frame. As positional data could not be reliably calculated, no further tests were performed using the Wii MotionPlus device. 


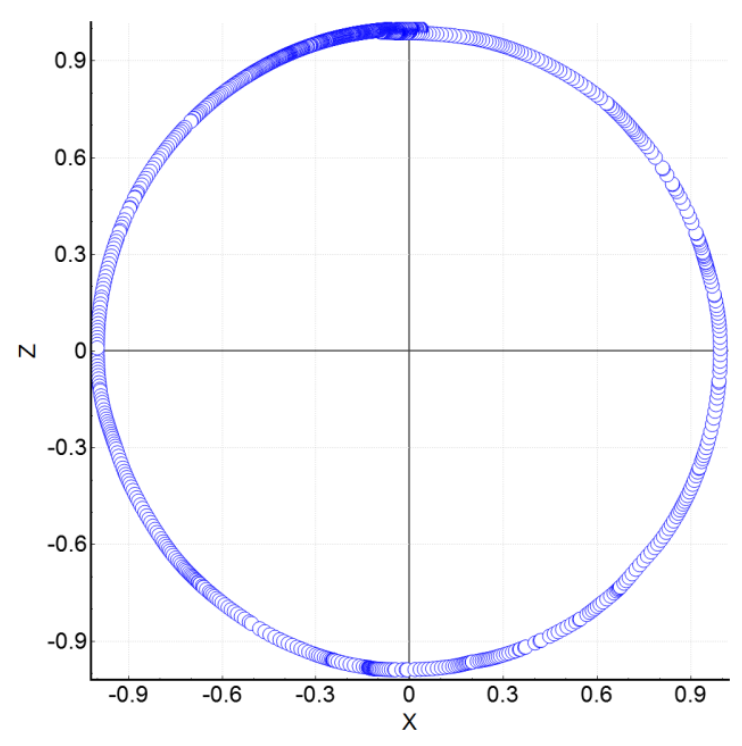

Figure 2: Wii orientation accuracy

\section{Kinect and Kinect2}

Trials of the Kinect devices reveal that both have practically identical performance at $2 \mathrm{~m}$ and $3 \mathrm{~m}$, however the Kinect2 benefits from updated optics and processing that allow it to track users at $1 \mathrm{~m}$ and $4 \mathrm{~m}$. Figure 3 illustrates this, with blue bars representing Kinect V2, and orange bars Kinect V1.

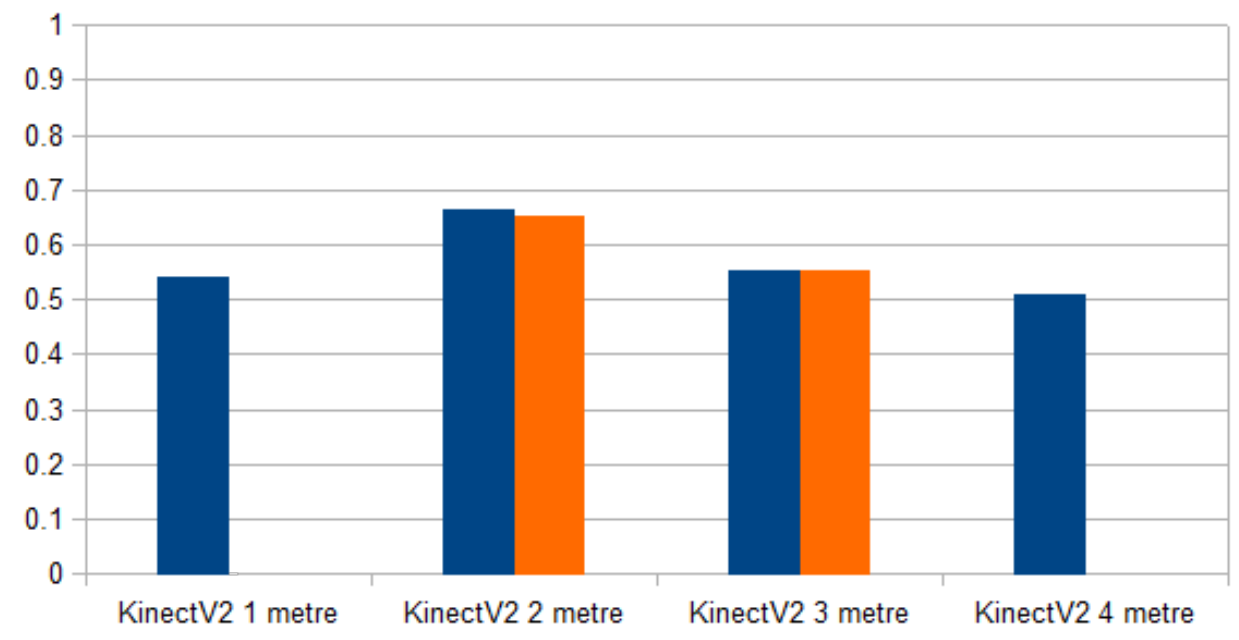

Figure 3: Kinect quality distance comparison

The Kinect scores at $2 \mathrm{~m}$ are noticeably lower than those of the other tested devices ( 0.65 for Kinect 1, 0.52 for Kinect 2, compared to 0.81 for PS MoveMe, and 0.85 for Sixense). Splitting up the quality metric on a per-axis basis in Table 4 clarifies where the weaknesses in the Kinect sensor lie.

\begin{tabular}{|c|c|c|c|}
\hline & Coronal & Transverse & Sagittal \\
\hline Kinect 1 & 0.839 & 0.204 & 0.807 \\
\hline Kinect 2 & 0.834 & 0.154 & 0.784 \\
\hline
\end{tabular}

Table 4: Kinect quality per axis at 2 metres 
Both Kinect devices display poor mean performance in the transverse plane (Kinect 1 quality 0.204 Stdev 0.23 , Kinect 2 quality 0.154 Stdev 0.143 ). This is unsurprising as rotations in this plane lead to the tracked hand becoming obscured. However, comparison with the MoveMe in the transverse plane (mean quality 0.68, Stdev 0.288), a similarly camera-based solution, reveals particular weakness in this plane.

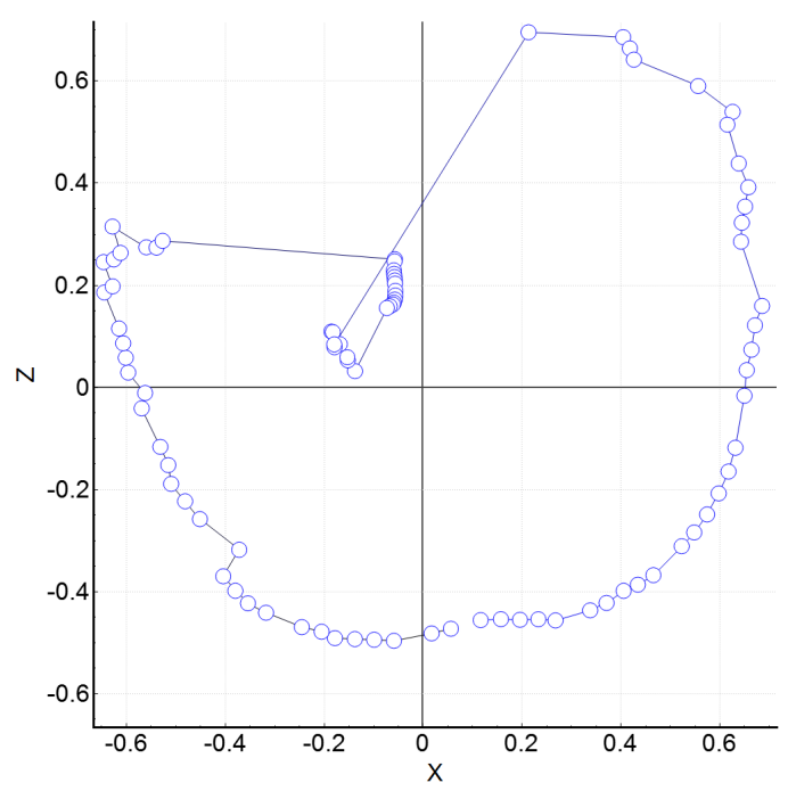

Figure 4: Plot of Kinect2 at 2 metres in transverse plane

The method whereby each device calculates its data must be considered. Both Kinects calculate an entire hierarchical skeleton of joint data, with the position of the wrist bone relying on the rest of the arm being correctly tracked, thus requiring more of the users arm to be visible than with the MoveMe, where the bright, uniquely coloured ball of the PS Move is found via simple computer vision techniques.

\begin{tabular}{|c|c|c|c|c|c|}
\hline & Axis & 1 metre & 2 metres & 3 metres & 4 metres \\
\hline \multirow{3}{*}{ Kinect 1 } & Coronal & - & 80.80 & 52.33 & - \\
\cline { 2 - 6 } & Transverse & - & 133.40 & 89.67 & - \\
\cline { 2 - 6 } & Sagittal & - & 62.40 & 47.00 & - \\
\hline \multirow{3}{*}{ Kinect 2 } & Coronal & 34.80 & 35.56 & 40.17 & 29.00 \\
\cline { 2 - 6 } & Transverse & 69.83 & 79.00 & 77.00 & 78.00 \\
\cline { 2 - 6 } & Sagittal & 36.00 & 31.57 & 34.50 & 38.50 \\
\hline
\end{tabular}

Table 5: Kinect sample count per axis

The quality scores of both Kinect devices are limited by the low sample rate of the devices. Kinect 2 in the transverse plane at $2 \mathrm{~m}$ is shown in Figure 4 . When compared to Figure 5 , the lower sample density becomes apparent (as shown in Table 5). The data also shows a phenomenon unique to the Kinect devices: as the user rotates such that their hand becomes obscured, the Kinect devices attempt to seek out the hand elsewhere in the image, frequently picking up the left hand or other part of the body, thus the cluster of data points towards the centre of the plot. This is in contrast to the PS MoveMe, which simply stops tracking until the ball is detected again. 


\section{Playstation Move}

During the PS MoveMe tests, the $1 \mathrm{~m}$ point was not successfully recorded, as the field of view of the camera was insufficient. Recorded orientation information was excellent, with orientation circularity of 0.95 in all 3 planes at $2 \mathrm{~m}$ and $3 \mathrm{~m}$, dropping to 0.76 at $4 \mathrm{~m}$. Unlike the other devices, orientation is derived from sensors on board the handheld device, rather than a camera image or magnetic field, and so is unaffected by distance. However a reduction in image position tracking leads to fewer orientation samples, impacting the circularity. Position circularity at $2 \mathrm{~m}$ is good (circularity 0.646 , stdev 0.178 ), but displays a rapid drop-off at distance.
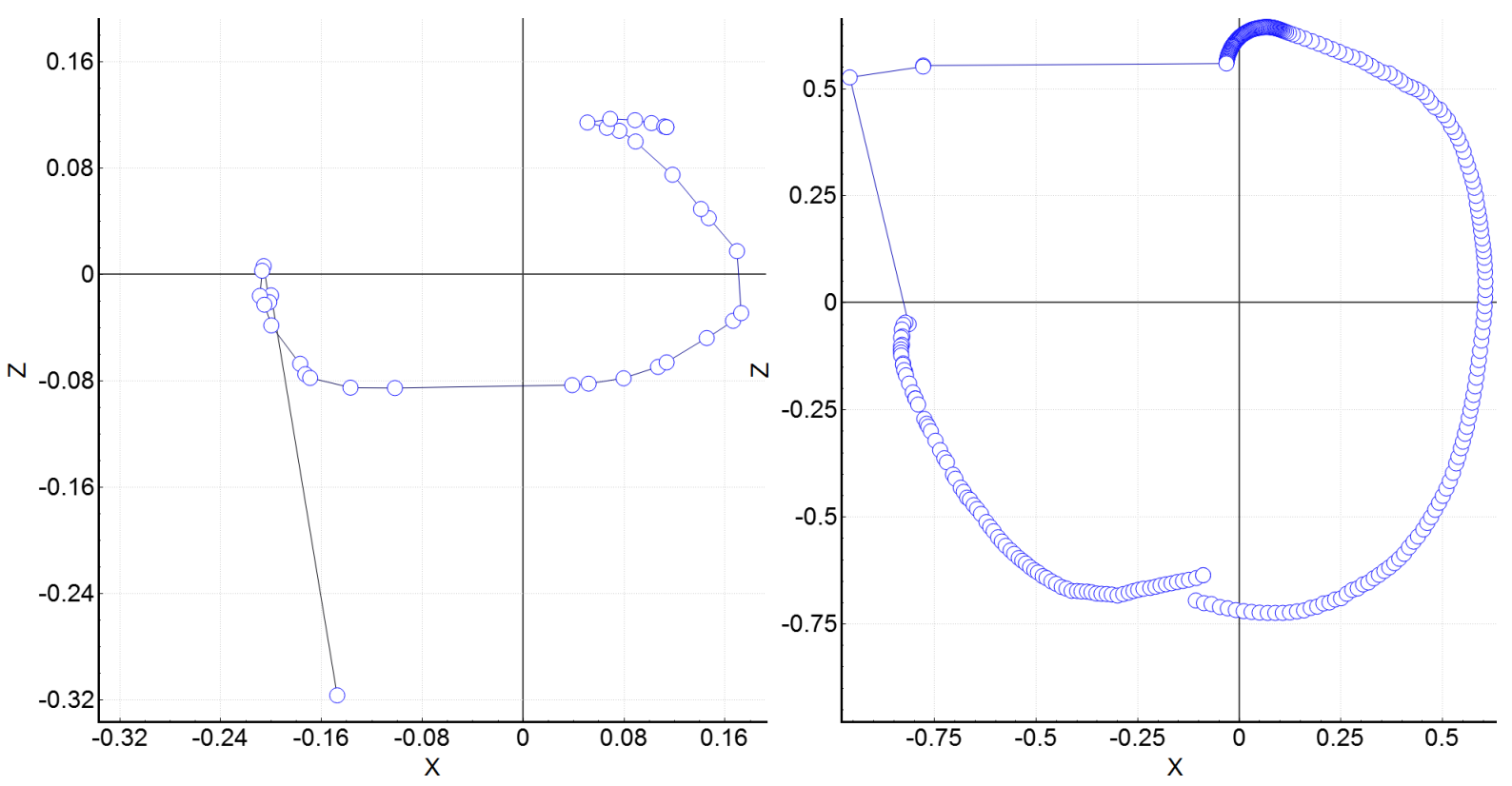

Figure 5: Plot of PS Move at $4 \mathrm{~m}$ and $2 \mathrm{~m}$ in transverse plane

Tracking of the PS MoveMe starts to degrade at $3 \mathrm{~m}$. Degradation of $\mathrm{z}$ axis tracking is most prominent, while movement in the coronal plane remains accurate. Figure 5 shows an example of the PS MoveMe in the transverse plane. Immediately obvious is the large gap in the top left quarter of the graph, with the beginning of the gap denoting the point at which the move was obscured, and the end the point at which tracking was regained, with an additional tracking fault. Data is also more ovular, with an approximately 2:1 ratio between minimum and maximum point lengths in the plot axis (position circularity: 0.45 ). This can be compared to the right hand plot, in which a transverse plane recording at $2 \mathrm{~m}$ shows an approximate $1: 1$ ratio (position circularity: 0.807 ). The position detection limit of the MoveMe software appears to be approximately $4 \mathrm{~m}$. It was noted that further movement in the $z$ axis caused a cut off effect, where no further movement in the axis is seen. 


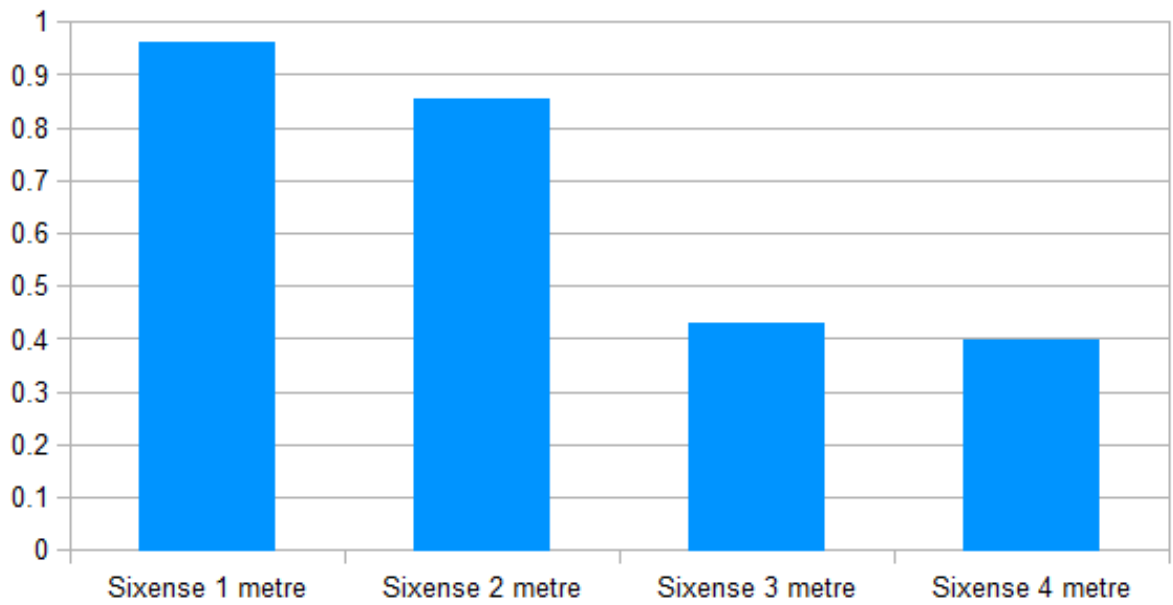

Figure 6: Sixense quality distance comparison

The Sixense is unique among the controllers as it can track position without the use of a camera. Its performance degrades quickly with distance, however. This can be seen in a graph of its combined score metric vs. distance, in which a combined score of 0.96 , drops by $50 \%$ at $3 \mathrm{~m}$ (Figure 6). This performance degradation is the worst over distance, and beyond $2 \mathrm{~m}$ the Sixense displays the worst quality metric of all devices. At a distance of $1 \mathrm{~m}$ (or even less, as the device is not constrained by a camera's field of vision) however, the device is the best performer, generating almost perfect circularity for both orientation and position.
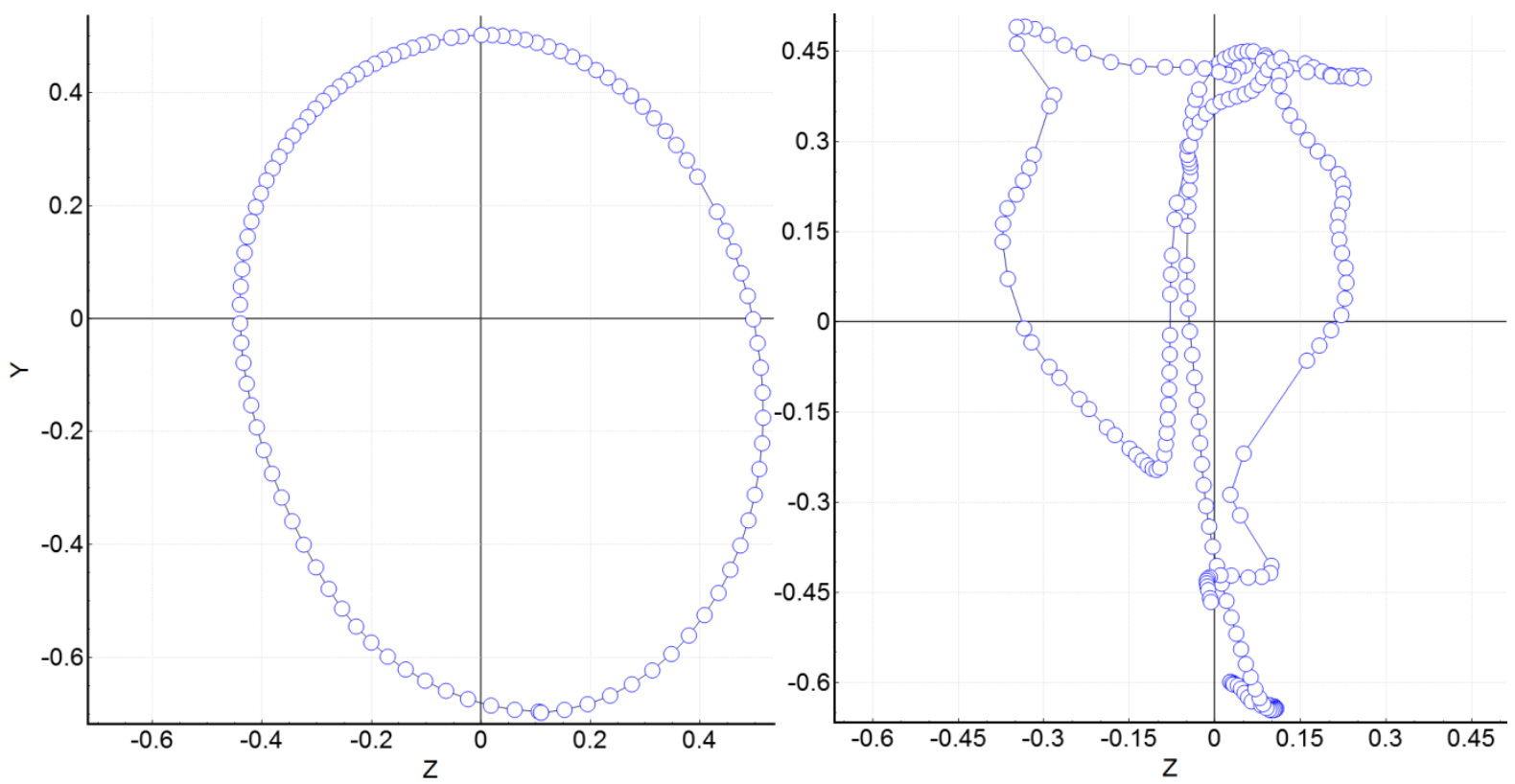

Figure 7: Plot of Sixense at $1 \mathrm{~m}$ and $4 \mathrm{~m}$ in sagittal plane

The results collated in Table 5 reveal a weakness in the sagittal plane. Table 6 shows the standard deviation of collected samples for each axis and distance. The quality of data extracted from the Sixense at $1 \mathrm{~m}$ has particularly low deviation in quality in the transverse plane. At $4 \mathrm{~m}$, the sagittal plane results in a consistently poor quality metric. The extent to which the Sixense degrades is best 
seen visually - Figure 7 shows recorded data in the sagittal plane at $1 \mathrm{~m}$ and $4 \mathrm{~m}$, with a breakdown in accuracy, making detection of a circular gesture impossible at the greater distance.

\begin{tabular}{|c|c|c|c|c|}
\hline Axis & $\mathbf{1 m}$ & $\mathbf{2 m}$ & $\mathbf{3 m}$ & $\mathbf{4 m}$ \\
\hline Coronal & 0.9592 & 0.9521 & 0.4361 & 0.6648 \\
\hline Transverse & 0.9847 & 0.9512 & 0.7023 & 0.3588 \\
\hline Sagittal & 0.9497 & 0.6622 & 0.1560 & 0.1740 \\
\hline
\end{tabular}

Table 6: Sixense circularity axis comparison

\begin{tabular}{|c|c|c|c|c|}
\hline Axis & $\mathbf{1 m}$ & $\mathbf{2 m}$ & $\mathbf{3 m}$ & $\mathbf{4 m}$ \\
\hline Coronal & 0.0257 & 0.0225 & 0.3634 & 0.3739 \\
\hline Transverse & 0.0064 & 0.0243 & 0.1657 & 0.2119 \\
\hline Sagittal & 0.0198 & 0.1837 & 0.2114 & 0.1075 \\
\hline
\end{tabular}

Table 7: Sixense quality deviation comparison per axis

\section{Recommendations}

Domestic motion capture devices have great potential for rehabilitative gaming. A benchmark for measuring the suitability of such devices has been presented. That benchmark has been applied to the commonly available systems and each has been shown to have strengths and weaknesses. Those relative strengths are now discussed and recommendations made as to how best to utilise the devices to the needs of a specific rehabilitative project.

Of note is the excellent quality of data obtained from the Sixense device when used at distances close to the base unit, showing high accuracy in both position and orientation across all three planes. This suggests the Sixense would make an excellent device to use in situations where a high degree of fidelity in movement detection is required, such as detection of specific limb movements and poses. The device begins to heavily degrade at distance, so careful considerations must be made as to the environment it is used in. Applications deployed via a laptop or tablet would be ideal conditions for the Sixense device.

The primary drawback of the PS MoveMe system is its reliance on expensive external hardware and a network connection, but in cases where greater working distances are required, the device produces quality positional and orientation information at $2 \mathrm{~m}$, with degradation in performance beyond that. At $2 \mathrm{~m}$, all trial participants could be fully detected with limbs outstretched, making the Playstation MoveMe a good choice for living room interaction on a larger screen. Further work will be required to determine whether tighter control of the environment, in regards to any patches of light within the PS MoveMe's field of vision, would result in improved performance at range.

Although the Kinects score less well, notably in the transverse plane, it should be pointed out that these devices provide data on the entire shape of the body. When used at a distance of $2 \mathrm{~m}$, with no hand occlusion, these devices produce quality data. At $2 \mathrm{~m}$, both Kinect devices are very similar, both displaying a weakness in recalculating body tracking sufficiently to determine hand position during rotations in the transverse plane. As with the MoveMe, further testing is required to determine the sensitivity of the Kinect to differing lighting conditions; however, several trials with the Kinect 2 showed the device tracking the left hand as the user turned away from the camera, suggesting that processing of the camera image for facial detection to infer orientation could have a positive effect on reducing false readings. 
It is hoped that these results will serve as a recommendation for future projects utilising domestic gaming devices for rehabilitation.

\section{Design and Development of a Gesture Based Video Game for Rehabilitation of Upper Limb after Stroke}

The development of a rehabilitative game for stroke patients is now described at length, focusing on the design choices made. The game was designed by a team of professional game developers and medical experts, and is intended to address the rehabilitation of arm movement in stroke victims.

A series of coordinated bi-manual movements have been identified as providing optimum and appropriate exercise for recovering stroke victims. These movements are used as the basis of the video game. The Sixense three-dimensional motion detection device is utilised to track the patient's upper limbs. The motion is used as the input for a professionally developed video game, which was designed specifically to utilise the prescribed exercises, and provides feedback on the patient's progress and recovery.

This section focuses on the implementation details which ensure that the game encourages the correct rehabilitative movements, and that it is sufficiently engaging to hold the patient's attention promoting compliance with the exercise regime. At each step of design and development, tenets from professional game development were incorporated into the overall medical goals of the project. Each of those tenets is discussed within the context of the game, and presented as advice for the development of serious games for health more generally. Conclusions and recommendations are drawn which can be utilised in the development of further teletherapy projects.

\section{Motivation for the Project}

Stroke is a major global health problem that is predicted to increase as the population ages (Murray 2010). Many stroke survivors suffer from hemiparesis, which is a partial paralysis of one side of the body caused by damage to the brain. Improvements to upper limb function can be achieved with intense, repetitive and challenging exercise of the affected limb (Langhorne 2009). A series of coordinated bi-manual movements have been identified which together form the functional basis for activities of daily living (Kimmerle 2003). Stroke victims suffering from impaired upper limb mobility are encouraged to perform these exercises on a daily basis to aid rehabilitation. However these exercises are, by their repetitive nature, time-consuming for the therapist and boring for the patient, which can result in less exercise being carried out than is recommended.

The use of video games to promote rehabilitative exercise has been suggested in numerous works, in order to keep the patient's attention and provide a more interactive experience (Davison 2014) (Rego 2010) (Flores 2008) (Saposnik 2011). Such an approach has a number of benefits: the game should provide a more involving context, so that the patient enjoys the rehabilitative process and is more likely to be engaged in it; the exercises can easily be carried out at home as gaming systems which include motion-capture devices are relatively commonplace and affordable; and data can be collected on the patient's recovery as the software monitors the patient's actions. Work to date on implementing such an approach has taken two distinct tacks -- either existing commercial games have been used as the basis of the study (Joo 2010) (Lange 2009), or bespoke games have been developed as part of the research project (Burke, Optimising engagement for stroke rehabilitation 
using serious games. 2009) (Burke, Serious games for upper limb rehabilitation following stroke 2009) (Morrow 2006) (Ushaw 2013). Utilising an existing commercial video game in this context has limitations as the game itself has not been developed with rehabilitation or monitoring in mind; the movements which are practised are those chosen by the game designers to most suit their gameplay, so are not necessarily the movements the medical professionals would choose to promote rehabilitation. Furthermore extracting data from a commercial game is impossible on a console, or without access to the source code and tool-chain on a PC. However, commercial games tend to be engrossing and immersive; factors which encourage the patient to persevere with the exercise. Conversely, the games developed specifically for the purpose of rehabilitation are designed to react to the prescribed exercise movements and can collate data pertaining to the patient's progress (Graziadio 2014). A game developed for rehabilitation offers the opportunity to include adaptive game-play, whereby the game detects when the patient is performing less well, and dynamically changes parameters of the game to suit the patient's abilities (Pirovano 2012) (Cameirão 2008) (Rossol 2011) (Borghese 2012). The downside of developing a bespoke application is that the quality of the game itself tends to be significantly lower than a commercial title for obvious budgetary reasons, so may not be as enjoyable and involving as a commercially developed game.

The project involved the development of a video game designed for rehabilitation of stroke-related upper limb disability, which is of sufficiently high calibre to be comparable to commercial games. The game uses three dimensional motion sensing technology to detect the movement of the patient's upper limbs, and presents the player with an ongoing series of challenges based on practising the coordinated bi-manual movements described in (Kimmerle 2003). The presentation of the game (graphics, animation, audio) is of a high quality, having been developed by a commercial games studio. A video game design ethos widely used in the games industry, which suggests that frequent in-game rewards encourages a player to remain engaged and continue playing (Koster 2013), is employed. This approach, while more costly, results in a game which requires the patient to practice the prescribed exercises and presents an interactive experience which encourages the patient to engage regularly in their home.

The overall aim of the project is to develop a validated system which provides health professionals with the capability of remote monitoring of home-based upper limb rehabilitation through interaction with a bespoke action video game. A widespread measure of upper limb rehabilitation is the Chedoke Arm and Hand Activity Inventory (CAHAI) score (Barreca 2004), which is calculated from a therapist's visual assessment of the patient carrying out a set of exercises. If this score can be predicted automatically from the motion capture data used by a game, as it is being played in the patient's home, then considerably more frequent monitoring can occur without the therapist having to be present.

\section{System Design}

The starting point for the design of the game was the range of moves which the patient must practice, so the first topic to consider is a discussion of why the moves were selected, and the devices and software which were used to detect them. The architecture of the system is also presented, focusing on the way in which the three-dimensional space is monitored, the progression of the exercise regime as the patient's dexterity improves, and the direct feedback that is provided to the patient while carrying out the exercises. The work involved in ensuring that the game feels as 
polished as a commercial release is also addressed, as this leads to an increased level of engagement by the patient.

\section{The Exercises}

It has been established that upper limb rehabilitation after stroke is promoted by frequent and repetitive exercise of the affected limb (Langhorne 2009). However it is also established that conventional rehabilitation programs carried out domestically, with no supervision from therapists, suffer from low compliance by patients (Touillet 2010). Anecdotally the patient is more likely to lose interest as the only direct feedback may be an increasing list of figures in a spreadsheet. Video games have been mooted as a source of greater compliance (Rego 2010), as feedback is more interesting (for example, higher scores and progress through challenges) so the patient remains engaged with the process of rehabilitation at an abstracted level.

In (Kimmerle 2003) a series of coordinated bi-manual movements of the upper limbs are identified as those which form the functional basis for daily living. The motions which were selected for the patient to perform while playing the action-video game comprise 100 separate upper limb patterns based on combinations of these movements. These identified movements are used to control the action in ten distinct mini-games, each with easy, medium and hard difficulty settings (based on the complexity of the movement required of the patient). The method whereby the progression of the games, and their rehabilitative movements, is fed back to the player is discussed later. The rehabilitative actions which were identified involve both movement of the upper limb through the three-dimensional space, and rotation of the wrist, elbow and shoulder joints around their axes of freedom (for example supination and pronation of the wrist, as well as flexion and extension). Consequently a motion tracking device was required which could reliably recognise these types of movement.

\section{The Motion Tracking Device}

As discussed earlier in this chapter, the choice of motion tracking device for a teletherapy project is vital. Key requirements when designing this game were a motion tracking device which would not only enable the tracking of the three-dimensional movement and rotation of limbs, but also include a SDK which allows complete control over the tracking algorithms, and access to the motion data which is recorded. This requirement of accessible data quickly ruled out the use of a home console, so the development platform was decided to be the PC. A comparison of the various affordable motion capture devices available for use with PC led to the decision that the Sixense was optimal for the project's purposes. Briefly stated, neither the Wiimote or the Kinect offer the three dimensional rotational input that was required, and the PS Move, while offering the full set of input data, requires an ungainly hardware combination of both a Playstation3 console and a PC in order to officially use the SDK. Further to this, as the application is intended to be used at relatively close quarter, via a laptop, the accuracy of motion detection at around 1 metre was vital.

The Sixense motion control system from Sixense Entertainment Inc. uses magnetic motion tracking to provide continuous position and orientation information. The use of electromagnetic fields is well established technology for reliably measuring three-dimensional space (Hansen 1987). Three controllers were used; one in each hand, and one tucked into the belt or pocket at waist level to provide further data on the patient's base position and motion. The base unit is located at shoulder height in front of the patient (but not blocking the game screen), so that it is in the centre of the full 
range of vertical upper arm freedom of movement. The distance between the patient and the base unit is also important, as magnetic field measurement is known to decay in strength and to distort as the distance between the source and sensor increases (Zachmann 1997). The optimal distance was determined to be around $60 \mathrm{~cm}$ (i.e. approximately an arm's length); if the sensors are detected to have gone beyond $80 \mathrm{~cm}$ from the detector, an on-screen message instructs the patient to move closer to the screen and base unit.

\section{The Game Design}

The game which was designed consists of a series of scenes involving circus performers carrying out their acts in a big top tent; the patient is instructed to perform specific move combinations and, if they succeed, the circus performer successfully completes the act. The more successful the acts that are performed, the more the player can progress through the game onto increasingly more complex moves.

Each circus act sequence is self-contained, and this modular nature offers some significant advantages. Importantly the game is structured in a way that the software knows which action the motion analysis algorithms are searching for at any time. A more generalised game wherein the patient may choose to perform any of a number of actions at a particular time would require considerably more complex motion analysis algorithms. The algorithms would need to test any player movement against the full range of possible exercises, which would take up a significant amount of the computing resources available on a standard laptop. Another key advantage arises as the order of the sequences can easily be changed, in response to play-testing by the initial test groups of patients. This means that movements which are found to be more difficult can be pushed further back in the progression, and easier movements can be brought forward. The standalone nature of each circus act also greatly aided the testing and bug-fixing phase of the project. As the player's actions can only influence one sequence at any time, there is limited opportunity for more complex system-wide issues to occur. Furthermore each sequence can attain a level of polish and professionalism independently of other sequences, leading to a more complete feeling to the game, which encourages the patient to engage for longer periods of time.

\section{Asymmetrical Motion Capture}

A key point to incorporate when developing a system for motion capture of the upper limbs of stroke victims is that the dexterity of the paretic limb is generally considerably less than that of the non-paretic limb. For example, if an exercise requires the patient to raise both arms as high above their head as possible, the patient is likely to be able to raise the unaffected limb to a significantly greater height than the limb requiring rehabilitation. Consequently the motion capture needs to be treated as asymmetric.

This asymmetry is achieved through the use of two distinct bounding volumes in the threedimensional space; one related to each arm. All movements are then interpreted in terms of percentages of those bounding volumes.

At the start of the first session with the game, the patient must go through a calibration process which defines the extent of each bounding box. The patient is instructed to reach as far as possible along each axis in turn; the process is repeated three times and the average reach taken as the extent of the bounding box along each axis. The bounding box dimensions for each limb are saved as part of the patient's profile for subsequent sessions. The patient also has the opportunity to 
recalibrate at the start of a subsequent session; this allows the therapist to recommend that the recalibration takes place as the patient has improved the reach of their paretic limb.

Each of the 100 rehabilitative moves is defined in terms of the percentile position within the bounding box of each Sixense controller, as well as the orientation of the controller. For example, a move may entail the movement of each limb between around $20 \%$ toward the lower vertical limit of the bounding box, and $60 \%$ toward the upper limit. For an able-bodied person these values will represent approximately equal distances for both arms, but for a recovering stroke victim, the distance for the paretic limb is likely to be significantly less. Consequently when tracking the motion of the sensors in three-dimensional space, it is the movement as a percentage of the bounding box dimensions which is used as a comparison. The move is declared successful if the measurements match the percentage-based parameters of the prescribed exercise. In that way, a patient with severely limited dexterity in one arm can still progress through the game and practice the exercises. Further note that this reduction in reach is not communicated by the in-game avatar which instructs the patient on which exercises to carry out. The avatar's reach remains constant, which encourages the patient to feel successful and to continue with the exercises.

\section{In-Game Help and Feedlback}

Visual aids are provided to instruct the player on the exercise which must be practised during each action sequence. These aids are presented as part of the game's graphical user interface (GUI) in real time. An avatar is displayed to the right of the screen, which is animated to show the movement that the patient is expected to perform in a sequence. The avatar repeats the motion throughout the game sequence. The avatar's hands are prominently coloured, whereas the rest of the avatar is plain white; this focuses the patient's attention on the movement of the hands which must be replicated during the exercise.

There are also two coloured rings displayed on the avatar in the GUI. These show the current position of the patient's hands - i.e. they represent the position information from the two magnetic sensors in the hands, relative to the sensor on the belt. The guide rings are colour-coded to match the avatar hand colours (green for left, blue for right - note that the avatar is a mirror image of the patient, as the patient faces the screen on which the avatar is displayed). It is important to note that the distance of the rings from the centre of the avatar is proportional to the full extent of the patient's bounding box. If the player's paretic limb is extended to its full ability, then the guide ring will be at the edge of the circular region in the GUI, in keeping with the approach we take of treating distances as percentages of the bounding boxes for each limb. This has the additional benefit of providing positive feedback to the patient, showing that their current full reach is acceptable for progress in the game.

It can be desirable for rehabilitative games to self-adapt to the patient's ability (Cameirão 2008) (Pirovano 2012). This entails monitoring the player's attempts at specific rehabilitative motions and, if the patient is repeatedly failing, to modify the parameters used in the detection routines to be more forgiving. For example (Pirovano 2012) uses Al algorithms to decide whether to allow more leeway in how much the feet are moving if a patient is struggling to progress. As the patients' ability is to be monitored remotely, a consistent set of parameters was required, so adaptive gameplay was not an option. The in-game help and feedback described in this section partly address this, coupled with the progression of difficulty in the sequences of moves from easier through to difficult. 
Further to the information provided by the avatar, we provide an option for the patient to view a video of a therapist carrying out the required rehabilitative movement. The patient may choose to watch this video at any time, and the game itself will pause while the video is played back on screen. If the patient has failed to meet the requirements of an action over a number of timed attempts, the option to view the explanatory video becomes more prominent in the GUI, encouraging the patient to study how to achieve the move without overt suggestion of requiring help or failure.

\section{Maintaining Player Compliance}

While there is much literature pertaining to the use of video gaming in therapeutic applications, the works tend to focus on either attempting to identify relevant uses of existing video games (Joo 2010) (Lange 2009), or have included a comparatively basic video game designed specifically for rehabilitation but developed by a research lab on a budget which is tiny compared to that of commercial games (Burke, Optimising engagement for stroke rehabilitation using serious games. 2009). While both approaches have yielded very interesting and promising results, the decision was taken early in this project to develop a video game of commercial quality which was designed specifically with rehabilitation in mind. The overall focus is to produce a game which will aid the long term recuperation of many patients, so it was felt that an appealing gaming experience was important.

The game involves high quality animations and music, as well as quirky and engaging characters. These serve the purpose of giving the rehabilitation sessions a sheen of fun and entertainment. The game could probably have been built solely around the basically coloured two dimensional avatar and the two guide-rings, but it was felt that this would not be a sufficiently engaging experience for the patient to keep coming back for repeated sessions. This level of polish comes at a price, of course; not only was the game considerably more expensive and time-consuming to develop, but the laptop itself must devote a significant proportion of its computing resources to the graphics and audio, leaving less computational power for the gesture recognition and data handling.

Various elements of mainstream game design have been utilised to encourage the patient to maintain compliance with the exercise regime. These are based on the idea that an enjoyable game presents the player with a continuous set of rewards and attainable challenges (Koster 2013). We include a series of "achievements", comparable to those on XBox or Steam; these are awarded when the player hits certain thresholds (for example, 50 hours of play, or successfully popping 100 balloons) and consist of a GUI element and a musical cue. The player can review achievements gained and those yet to attain from the menu screens. We also employ a high score table, for each challenge, so the player can see progress and aim for improvement. The scores are based on the number of moves successfully completed during a mini-game (i.e. there is no gradation of success of any particular move). These elements combine to encourage the patient to continue with the exercises and to sustain a level of abstraction between playing the game, and carrying out repetitive exercise.

\section{Implementation and Evaluation}

The game was developed as a system to provide remote monitoring by health professionals of home-based upper limb rehabilitation. A part of the purpose of this study was to derive clinically relevant measures of upper limb function from analysis of the movements made by patients while 
playing the game. Some requirements of that work have direct bearing on the implementation so are discussed in this section to provide guidance on developing a game with medical professionals.

\section{Assessment Build}

The game is required to gather data on the patient's movement for analysis. This is achieved through an assessment build of the game. The assessment build consists of a sequence of 12 to 40 exercises which the patient plays through one after the other, with no retries of failed exercises, or choices about difficulty or ordering. The raw data provided by the Sixense motion control system is recorded and packaged for analysis; i.e. no real-time analysis or filtering of the data takes place. In effect, the patient uses the main build of the game as the rehabilitative aspect of the process, practising the various exercises, and the assessment build is then used to feedback progress to the medical practitioners.

The first task for the patient during an assessment is the calibration process. The patient is instructed to carry out a series of motions which record the extent of the reach of each limb and where the mid-points are, in all three dimensions. It is important to start each assessment with this calibration as the patient's reach may have improved since the previous assessment. The patient is then instructed to attempt each of the 12 to 40 assessment moves in turn. The raw data from each of the sensors (position and orientation) is stored throughout the exercises. No filters are applied, and the data is not interpreted in terms of percentages of the bounding boxes, so that any assumptions made in processing the input stream when attempting to recognise moves do not colour the analysis. If the player fails to achieve an action within the time limit, the data is still recorded and the game moves on to the next exercise.

When the assessment is complete, the full data is packaged, and labelled with a unique ID for the patient and the date. It can then either be collected by a therapist, or uploaded automatically to the cloud, for analysis. The movements which comprise the assessment build have been chosen as those which give the therapists the most information about the progress of the patient's rehabilitation. They are not necessarily the moves which most contribute toward that rehabilitation. The assessment moves are distributed throughout the main version of the game, along with all the other rehabilitative moves.

\section{Ordering the Moves}

The rehabilitative actions which the patient is asked to perform are split into three categories: easy, medium and difficult. Furthermore, within these categories there is a progression as more complex moves are introduced. The initial ordering was defined according to the therapists, but extensive play-testing from patients revealed that some of the moves were incorrectly rated in terms of the challenge they presented. This required the order of the moves to be changed in later builds of the game. The system architecture has been developed to be both modular and data-driven. This meant that reordering the moves simply involved changing the ordering in a data file: this is a common approach in well-constructed video game architectures. The exact content of the assessment build is also governed by a data file, so again it can be changed with ease as requirements evolve over the period of study.

The overall structure of the game is governed by a series of levels which are gradually unlocked as the patient progresses through the rehabilitative exercises. Initially, for example, only the easiest challenges are available; completing an easy challenge causes a more difficult challenge to become 
available. A level selection screen is also included. Again this is a fairly standard implementation within a video game; however it has additional benefits in terms of rehabilitation. Presenting the player with only a few exercises to begin with encourages focus on those exercises which are initially most therapeutic and achievable, without overwhelming the new patient with too many options. Also, presenting the exercises as a series of levels which must be attained through success in earlier challenges gives the patient a series of goals to aim for. In gaming, it is important to provide a player with a constant stream of rewards, in order to maintain interest, and a classic example is unlocking extra levels as the game progresses (Koster 2013). This encourages the patient to not only continue with the exercises that have already been practised but to strive toward more complex exercises.

\section{Identifying the Paretic Limb}

The exercises are designed to exercise the paretic limb; in many instances this entails different movements for the non-paretic limb. However the affected arm could be either the left or right, so the software must take this into account. The GUI must show the avatar moving each limb appropriately and the motion recognition algorithms must track the correct limb for each part of the exercises.

At the start of the calibration process, the patient is instructed to hold controller 1 in the good hand, to hold controller 2 in the hand with limited dexterity, and to clip controller 3 to their belt. The Sixense system uses a set of LEDs on each controller to show how they are numbered (based on the order in which they were detected at system start). The software then detects whether the controller in the paretic limb is to the left or to the right of the controller in the belt and in the nonparetic limb. Once this is established, the detection algorithms track the relevant hand for each half of the exercises - i.e. we consider the two limbs, and their associated bounding boxes, as the paretic and non-paretic limbs, rather than the left and right limbs. Also, if necessary, the graphics in the GUI for the avatar are flipped around the vertical axis. As the GUI consists of two-dimensional textures in an orthographic projection, this is a simple matter of reflecting the texture coordinates around the vertical centre of the texture maps.

\section{Patient Profile Data}

The game saves profile data for each patient that plays it. This data serves two functions related to game-play dynamics and ease-of-use, and to patient monitoring.

The first type of data is that related to the player's parameters and progress. So that the patient does not have to go through the calibration process every time the game is played, the parameters of the bounding boxes are saved to the profile and reloaded on subsequent exercise sessions (the patient may use the application several times a day, and is more likely to do so if the exercises can be accessed directly with minimal set-up time). The profile also includes an indication of whether the paretic limb is the left arm or the right arm. Additionally game progress data is saved, which includes a list of whether each level has been unlocked in the progression, values pertaining to the achievements, and preferences on audio volume, brightness of the screen, etc.

A further set of profile data is generated for each session which contains details of the patient's progress and engagement. This data consists of information such as how long, and how often, each exercise was practised, the success rates of the exercise attempts, and the scores attained. This data is collected by the therapist for further consideration, and is clearly marked with unique identifiers 
for the patient and date. Further to this, the raw data from the Sixense input device is saved to a set of data files for further analysis by researchers on the project if required.

\section{Conclusions Drawn from the Project}

The development of a video game for rehabilitation of upper limb dexterity in stroke victims has been described in detail. The section has focused on the aspects of the technology and the design which contribute toward the game's rehabilitative nature, both in terms of providing the medically relevant exercise and feedback, and presenting an entertaining and encouraging medium for that exercise. The game utilises readily available motion capture technology and runs on a standard laptop, so that it can be used as often as the patient likes within the home.

The game was designed to incorporate the specific exercises that have been identified as contributing to upper limb rehabilitation, and it was developed to a commercial standard. This combination is intended to provide a sufficiently entertaining experience for the target patients so that they persevere with the course of exercises. The motion capture algorithms were designed specifically to monitor the asymmetrical upper limb movement exhibited by stroke survivors. The instructions given by the game, and the feedback on patient progress, are couched in familiar video game graphical user interface elements, and are also presented in a way which does not penalise, or belittle, patients with more severe dexterity issues. This is achieved through the use of a separate bounding box for each limb, and measuring all movement as a percentage of the extents of the bounding box, rather than as absolute values. Familiar game design elements are also employed to create a progression of difficulty, and reward, as the patient's dexterity improves over the course of time.

The game embeds its rehabilitative exercises within a charming and friendly context of circus performances, utilising state-of-the-art computer graphics, animation and audio. Anecdotal reportage from the patient test group shows that the game is successfully encouraging more exercise, and therefore higher compliance with the rehabilitative program.

\section{Recommendations for Commodity Game Technology in Teletherapy}

The chapter has addressed the use of commodity video game technology in teletherapy. The issues have been discussed in a practical manner relating them to the development of a game for rehabilitation of stroke, and to a benchmark for motion capture input devices. A number of recommendations can be made from these studies, which can eb applied to future projects applying serious gaming to teletherapy.

The choice of input device is of paramount importance in designing a serious game for rehabilitation. The benchmark described in this chapter has been applied to each of the currently commercially available devices, and each has been shown to have its own advantages.

- The Playstation Move system demonstrates highest fidelity at range of two to four metres, making it an ideal option for a game played on the television in a family room environment. The disadvantage of this system is that it requires both a Playstation console and a PC to operate in tandem.

- The Kinect motion capture system performs reasonably well at around two to three metres, and the Kinect2 extends this range to between one and four metres. While the fidelity is not 
as that measured for PS Move, the Kinect systems have many additional features (face recognition, heart-beat monitoring, full skeletal tracking) which may be of interest in a specific teletherapy project.

- The Sixense motion sense controllers have the highest fidelity at closer distances (up to two metres), making them the ideal choice for a laptop based application where the patient is closer to the screen. Further to this, as the Sixense uses magnetic fields, there are no issues with line of sight to the controllers (which mars the functionality of the other systems considered).

A rehabilitative game should be developed with both the medical requirements in place and the need for the game to be sufficiently involving that the patient makes maximum use of it. In other words, if the user is sufficiently entertained to regard the process as a game rather than a rehabilitative program, then the chances of continued engagement are increased.

- Feedback to the player should imply success. In the project described in this chapter, a patient with stroke does not necessarily have the full reach of the paretic limb. This is not reflected in the avatar or the instructions - if the patient is reaching as far as possible, then the avatar should show complete success.

- A modular game design has multiple advantages

- It is easier to change the order of levels or challenges, depending on difficulty testing or individual patient requirements

- It is a less complex task to bring each level or challenge up to a high quality level, and to fix bugs, without affecting other sections of the game

- Assessment of each challenge can lead to removing the poorly performing ones, or identifying which need more work, without affecting the overall game

- As much in-game help and feedback as possible is of high importance. If a patient is struggling to meet a challenge, or has forgotten what to do, the instruction s should be readily available, on screen, and simple to follow. Incorporating videos of the actions being carried out can be a useful way of implementing this

- The game itself should be entertaining and involving, animations and sound effects should be amusing. Engaging professional game developers is a route toward this.

- Commercial games keep player interest with rewards. This can be replicated in a teletherapy project. Methods of implementing this include high score tables, achievements and daily challenges.

- Minimal set up time is also important. A patient is more likely to engage if the game is "plug and play". In particular the game should remember the patient's details so that progress can continue from one session to another without a lengthy configuration process.

Finally it should be noted that games in teletherapy can be utilised to gather a vast amount of raw data on the patient's state and progress. Real time gaming allows this data to be uploaded to the cloud, or otherwise collected for further analysis.

It is hoped that this chapter will serve as a guideline to the development of gaming projects for teletherapy. The recommendations made, while based on actual projects, are intended to be applicable generally. Many design choices must be made when developing a game that will provide some form of rehabilitation or monitoring. These choices must ensure that the game will both 
provide the medical intention and engage the potential users to the point where they regard themselves as players rather than patients during the process.

\section{Bibliography}

Barreca, S., Gowland, C. K., Stratford, P., Huijbregts, M., Griffiths, J., Torresin, W., ... \& Masters, L. "Development of the Chedoke Arm and Hand Activity Inventory: theoretical constructs, item generation, and selection." Top Stroke Rehabil, 2004: 31-42.

Borghese, N. A., Pirovano, M., Mainetti, R., \& Lanzi, P. L. "An integrated low-cost system for at-home rehabilitation." 18th International Conference on Virtual Systems and Multimedia. IEEE, 2012. 553-556.

Burke, J. W., McNeill, M. D. J., Charles, D. K., Morrow, P. J., Crosbie, J. H., \& McDonough, S. M. "Optimising engagement for stroke rehabilitation using serious games." The Visual Computer, 2009: 1085-1099.

-. "Serious games for upper limb rehabilitation following stroke." Conference in Games and Virtual Worlds for Serious Applications. IEEE, 2009. 103-110.

Cameirão, M. S., Bermudez i Badia, S., Oller, E. D., \& Verschure, P. F. "Using a multi-task adaptive VR system for upper limb rehabilitation in the acute phase of stroke." Virtual Rehabilitation. IEEE, 2008. 2-7.

Davison, R., Graziadio, S., Shalabi, K., Ushaw, G., Morgan, G., \& Eyre, J. “Early response markers from video games for rehabilitation strategies." ACM SIGAPP Applied Computing Review 3, no. 14 (2014): 36-43.

Flores, E., Tobon, G., Cavallaro, E., Cavallaro, F. I., Perry, J. C., \& Keller, T. "Improving patient motivation in game development for motor deficit rehabilitation." Proceedings of the 2008 International Conference on Advances in Computer Entertainment Technology. ACM, 2008. 381-384.

Graziadio, S., Davison, R., Shalabi, K., Sahota, K. M. A., Ushaw, G., Morgan, G., \& Eyre, J. A. "Bespoke video games to provide early response markers to identify the optimal strategies for maximizing rehabilitation. ." Proceedings of the 29th Annual ACM Symposium on Applied Computing. ACM, 2014. 20-24.

Hansen, Per K. Method and apparatus for position and orientation measurement using a magnetic field and retransmission. USA Patent 4,642,786. 10 February 1987.

Joo, L. Y., Yin, T. S., Xu, D., Thia, E., Chia, P. F., Kuah, C. W. K., \& He, K. K. "A feasibility study using interactive commercial off-the-shelf computer gaming in upper limb rehabilitation in patients after stroke." Journal of rehabilitation medicine, 2010: 437-441.

Kimmerle, M., Mainwaring, L., \& Borenstein, M. "The functional repertoire of the hand and its application to assessment." American Journal of Occupational Therapy, 2003: 489-498.

Koster, R. Theory of fun for game design. O'Reilly Media, Inc, 2013. 
Lange, B., Flynn, S., \& Rizzo, A. "Initial usability assessment of off-the-shelf video game consoles for clinical game-based motor rehabilitation." Physical Therapy Reviews, 2009: 355-363.

Langhorne, P., Coupar, F., \& Pollock, A. "Motor recovery after stroke: a systematic review." The Lancet Neurology, 2009: 741-754.

Morrow, K., Docan, C., Burdea, G., \& Merians, A. "Low-cost virtual rehabilitation of the hand for patients post-stroke." International Workshop on Virtual Rehabilitation. IEEE, 2006. 6-10.

Murray, C. J., Vos, T., Lozano, R., Naghavi, M., Flaxman, A. D., Michaud, C., ... \& Bridgett, L. “ Disability-adjusted life years (DALYs) for 291 diseases and injuries in 21 regions, 1990-2010: a systematic analysis for the Global Burden of Disease Study." The Lancet, 2010: 2197-2223.

Pirovano, M., Mainetti, R., Baud-Bovy, G., Lanzi, P. L., \& Borghese, N. A. "Self-adaptive games for rehabilitation at home." IEEE Conference on Computational Intelligence and Games . IEEE, 2012. 179-186.

Rego, P., Moreira, P. M., \& Reis, L. P. "Serious games for rehabilitation: A survey and a classification towards a taxonomy." 5th Iberian Conference on Information Systems and Technologies . IEEE, 2010. 1-6.

Rossol, N., Cheng, I., Bischof, W. F., \& Basu, A. " A framework for adaptive training and games in virtual reality rehabilitation environments." Proceedings of the 10th International Conference on Virtual Reality Continuum and Its Applications in Industry. ACM, 2011. 343346.

Saposnik, G., \& Levin, M. "Virtual reality in stroke rehabilitation a meta-analysis and implications for clinicians." Stroke, 2011: 1380-1386.

Smisek, J., Jancosek, M., \& Pajdla, T. "3D with Kinect." In Consumer Depth Cameras for Computer Vision , 3-25. Springer, 2013.

Touillet, A., Guesdon, H., Bosser, G., Beis, J. M., \& Paysant, J. "Assessment of compliance with prescribed activity by hemiplegic stroke patients after an exercise programme and physical activity education. ." Annals of physical and rehabilitation medicine, 2010: 250-65.

Ushaw, G., Ziogas, E., Eyre, J., \& Morgan, G. "An Efficient Application of Gesture Recognition from a 2D Camera for Rehabilitation of Patients with Impaired Dexterity." HEALTHINF. INSTICC, 2013. 315-318.

Zachmann, G. " Distortion correction of magnetic fields for position tracking." Proceedings of Computer Graphics International. IEEE, 1997. 213-220. 